\title{
System-level simulation of a self-powered sensor with piezoelectric energy harvesting
}

\author{
Loreto Mateu and Francesc Moll \\ Universitat Politècnica de Catalunya (UPC) \\ Department of Electronic Engineering \\ Campus Nord, Edifici C4, C/ Jordi Girona 1-3, 08034 Barcelona, Spain \\ $\{$ mateu,moll $\} @$ eel.upc.edu
}

\begin{abstract}
This paper presents a complete system simulation of a self-powered communication module. The components are described with the Verilog-A language, that allows to merge the electrical and mechanical models of the system. The self-powered sensor system is composed by an energy harvesting piezoelectric generator that powers a $R F$ transmitter. The simulations here presented compare between the case of a battery-less and battery-powered system. The results obtained with the simulation model implemented allow to show how design choices of the system change the periodicity of the transmission and the ability to recharge the battery.
\end{abstract}

\section{Introduction}

Since the beginning of the electronic industry, a continuous trend of size reduction and functionality increase has been observed. This trend has allowed the design of very small and powerful sensors that are able to transmit the data to a central receiver or to other sensors, forming what is known as sensor networks.

One particular area of application of these small sensors is the measurement of some property related to a person, like acceleration or medical parameters. For these applications it has been proposed that energy coming from the user activity may be harvested [10] to feed a network of very low power sensors, e.g. Body Area Network (BAN) and Personal Area Network (PAN) [11].

The area of energy harvesting has been active since the late 1990s with pioneering work by Starner [9] and Paradiso at MIT on piezoelectric films inserted on shoes [4]. Other methods to harvest energy from the environment have been proposed and investigated. During the last years a lot of advances in modeling of these energy sources have taken place.

From the point of view of the application, it is necessary a model of the complete system, preferably integrated in a standard CAE environment, to optimize the design of the complete system. The optimization of the separate components is not possible considering them independently because all the parts of the system (energy source, power conditioning, and load energy consumption) interact with each other. Some laboratories proposed to model a piezoelectric generator employing Verilog-A or VHDL-AMS to connect it to the electrical blocks of the self powered system, presenting the results obtained from a piezoelectric generator model done in VHDL-AMS [1]. The electrical part of the self powered system presented was also modeled in VHDLAMS to allow the simulation of the complete system in the same environment, thus decreasing the simulation time compared to a simulation made in a mixed-environment.

This paper presents a simulation environment based on the high level mixed signal modeling language VerilogA [8]. This language is the analog subset of the VerilogAMS language, and it allows the description of both electrical or non-electrical components, allowing a whole energy harvesting system to be described and subsequently simulated with a standard simulator. The objective of this paper is to pcreate a simulation environment that combines mechanical and electrical models involved in energy harvesting applications. The piezoelectric transducer mechanical model and the electrical components model (rectifier, voltage regulator, RF transmitter, battery) of the self-powered sensor system allow to evaluate the feasibility of different possible scenarios (e.g. RF energy consumption profiles, or battery versus battery-less operation).

The structure of the paper is divided in the following sections. The description of the simulation components is made in section 2. Section 3 shows several simulations of the time domain model considering two examples: a sensor with RF transmission and the charging of a battery. Finally, 
section 4 presents the main conclusions of the paper.

\section{Structure of a self-powered system}

A self-powered system based on environment energy harvesting is composed generically of several components (Figure 1):

- Energy transducer, to convert some available energy source into electrical energy. The environmental energy sources available for conversion may be thermal (thermoelectric cells), light (photovoltaic cells), RF (rectifying antennas), and mechanical (piezoelectric, magnetic induction, electrostatic converters).

- Storage capacitor. Some of the above energy transducers do not provide DC current, and in that case it is necessary to rectify the current and accumulate the energy into a capacitor.

- Voltage regulator, to adapt the voltage level to the requirements of the powered device.

- Battery, depending on the requirements of the application. In some applications the powered device can be completely switched off during certain intervals and a battery is not necessary, while in others a permanent powering is mandatory.

- Electronic device, with varying power requirements depending on the application.

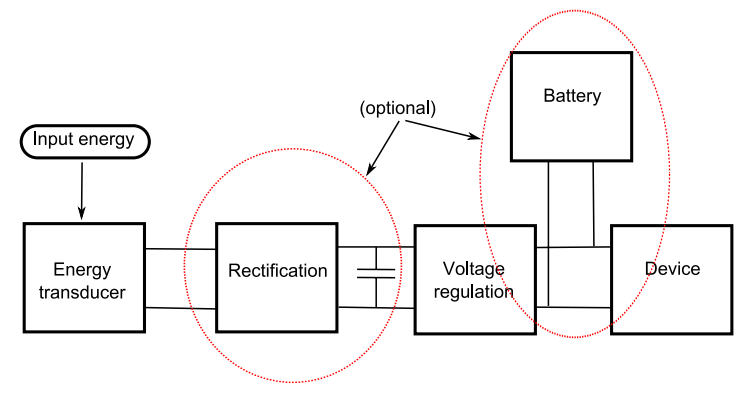

Figure 1. Schema of a generic self-powered device.

In this work, to illustrate the simulation environment, we present the case of a piezoelectric film inserted inside a shoe. In this case, the storage capacitor and rectification are necessary because each bending of the film produces a succession of alternating current peaks. In addition, being the energy source discontinuous, it is necessary to connect the load only when there is enough energy stored in the capacitor. Next, the description of each component is presented.

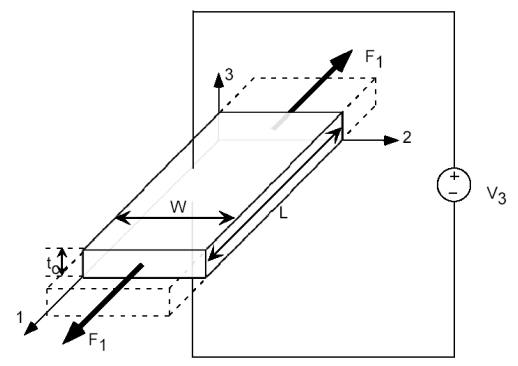

\section{Figure 2. Mechanical excitation of the piezo- electric film along axis 1.}

\subsection{Piezoelectric film}

The piezoelectric effect is a property of certain materials, that when subjected to mechanical strain, suffer an electrical polarization proportional to the applied strain. The phenomenon of piezoelectricity is described by the piezoelectric constitutive equations:

$$
\begin{aligned}
& S_{i}=s_{i j}^{E} T_{j}+d_{l i} E_{l} \\
& D_{m}=\varepsilon_{m n}^{T} E_{n}+d_{m k} T_{k} \\
& \text { for } i, j, k=1, \ldots, 6 \text { and } l, m, n=1,2,3
\end{aligned}
$$

where $T$ is the applied mechanical stress $\left[\mathrm{N} / \mathrm{m}^{2}\right], E$ is the applied electric field [N/C], $d$ corresponds to the piezo strain $\left[\left(\mathrm{C} / \mathrm{m}^{2}\right) /\left(\mathrm{N} / \mathrm{m}^{2}\right)\right], \varepsilon^{T}$ is the permittivity $[\mathrm{F} / \mathrm{m}]$ under conditions of constant stress, $D$ is the electric displacement $\left[\mathrm{C} / \mathrm{m}^{2}\right], S$ is the mechanical strain $[\mathrm{m} / \mathrm{m}]$, and $s^{E}$ is the compliance tensor $\left[\mathrm{m}^{2} / \mathrm{N}\right]$ under conditions of constant electrical field.

Following this behavior, a piezoelectric generator electrical model consists in a charge source in parallel with a capacitor.

The selected piezoelectric material is a PVDF film. These films are only metalized in the plane perpendicular to direction 3, so that $D_{1}=D_{2}=0$. The mechanical excitation is along axis 1 (orthogonal to film surface), and external applied electric filed is zero for the generator. Equation (2) expresses the electric displacement generated in the piezoelectric element under these conditions. The mechanical stress is related to the strain $S_{1}$ by Young's modulus $Y_{11}$

$$
D_{3}=d_{31} T_{1}=d_{31} Y_{11} S_{1}
$$

Given that the charge is given by the film area times the electric displacement, $Q_{3}=D_{3} A$ :

$$
Q_{3}=d_{31} Y_{11} S_{1} A
$$




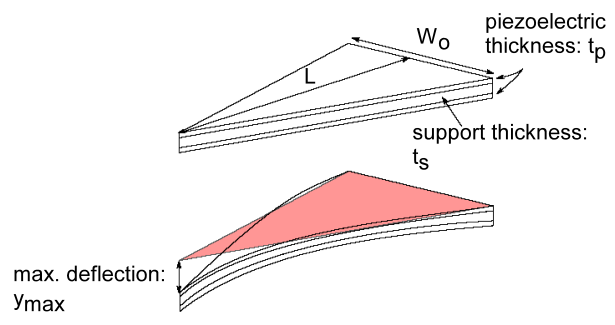

Figure 3. Structure of the piezoelectric cantilever.

\subsubsection{Triangular cantilever structure}

One convenient method to cause a mechanical excitation in the 1 axis is to use a cantilever structure with a vertical structure as shown in Figure 3, consisting in two piezoelectric films attached (each of thickness $t_{c} / 2$ ) to the top and bottom surfaces of a support material of thickness $t_{s}$. It can be shown that for a given vertical force, a maximum strain is obtained for a triangular shape. The expression of strain in function of vertical deflection can be calculated [5] for the structure shown in Figure 3, giving a charge related to deflection $y$ :

$$
Q_{3}=y \frac{W_{0} t_{c}}{2 L} d_{31} Y_{11}(1+2 \tau)
$$

where $\tau=t_{s} / t_{c}$ is the ratio of piezoelectric film thickness to support material thickness. On the other hand, the value of piezoelectric capacitor, $C_{p}$, is:

$$
C_{p}=\frac{4 \varepsilon_{33}^{T} W_{0} L}{t_{c}}\left(1-k_{31}^{2}\right)
$$

Finally, for varying deflection in time, a current is produced by the piezoelectric film, as:

$$
i_{p}=\frac{d Q_{3}}{d t}=\dot{y} \frac{W_{0} t_{c}}{2 L} d_{31} Y_{11}(1+2 \tau)
$$

These expressions are included in the piezoelectric Verilog-A model. In our example, the piezoelectric element is placed inside the insole of a shoe. The mechanical walking activity cause a mechanical deflection on the piezoelectric films. From voltage measurements on the piezoelectric films, it has been deduced the waveforms that appear in Figure 4. Thus, the maximum deflection, $y_{\max }$, applied at $x=L$ over the cantilever beam is taken as a triangular waveform with $t_{z 1}=110 \mathrm{~ms}$ rise time, $t_{z 2}=80 \mathrm{~ms}$ fall time, $A m p l=3 \mathrm{~mm}$, and a period of $T=400 \mathrm{~ms}$, see Figure $4 a)$. Figure $4 b$ ) is deduced from Figure 4a) employing Equation 6 and 4.

The dimensions and the constants of the piezoelectric films employed are: $W_{0}=30 \mathrm{~mm}, L=30 \mathrm{~mm}, t_{c} / 2=$
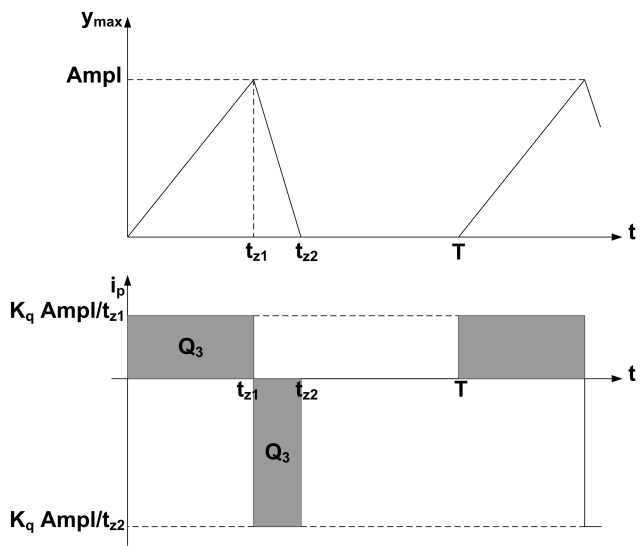

Figure 4. Maximum deflection, $y_{\max }$, applied to the piezoelectric film and generated current waveform, $i_{p}$.

$28 \mu \mathrm{m}, t_{s}=1 \mathrm{~mm}, y_{\max }=3 \mathrm{~mm}, Y_{11}=3 E 9 \mathrm{~N} / \mathrm{m}^{2}$, $k_{31}=0.12$, and $d_{31}=23 E-12 \mathrm{C} / \mathrm{N}$. In order to increase the generated current, a parallel electrical connection of the cantilevers is considered.

\subsection{Storage capacitor}

The energy needed to power the electronic device, $E_{r e q}$, employing a linear regulator is accumulated in a storage capacitor, $C_{s}$. The circuit operation is as follows. As the piezoelectric element is repetitively stressed, the voltage on the storage capacitor, $C_{s}$, increases. Whenever this voltage reaches a certain limit $\left(V_{o n}\right)$, the capacitor is connected to the regulator input by the control circuit, and it is discharged down to a lower voltage $\left(V_{o f f}=V_{d d}+\epsilon\right)$ that ensures a proper operation of the control circuit and the regulator, providing the energy required by the load $\left(E_{r e q}\right)$.

$$
E_{r e q}=\frac{1}{2} C_{s}\left(V_{o n}^{2}-V_{o f f}^{2}\right)
$$

The number of steps needed to reach $V_{o n}$ obviously increases with the value of $V_{o n}$. On the other hand, the value of capacitor $C_{s}$ and the voltage interval $\left(V_{o n}^{2}-V_{o f f}^{2}\right)$ are related by the required energy. An optimum value of capacitor can be found relating these magnitudes [6].

\subsection{Voltage conversion/regulation}

The voltage regulator can be modeled at a behavioral level as an element that provides a constant voltage when the input voltage surpasses a certain level. Then, the load drains a certain amount of current. This current, plus an additional ground current of the regulator is the input current 
that must be provided by the storage capacitor and piezoelectric film. Then, the behavioral model can be described schematically as shown in Figure 5.

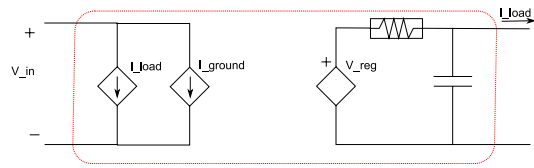

Figure 5. Behavioral model of the voltage regulator.

As the voltage regulator drains current from the storage capacitor, its voltage starts to decrease. Therefore, hysteresis needs to be implemented to avoid continuous ON/OFF oscillations of the regulator output.

\subsection{Battery}

A recently published battery model developed by Chen et al. is used in this paper, capable of predicting runtime and transient response [2]. Whenever the voltage regulator is connected, there will be a transfer of energy to the battery. Figure 6 shows the schema of the model, that is also implemented in Verilog-A. The values of the parameters were taken from [2] corresponding to a polymer Li-ion battery.

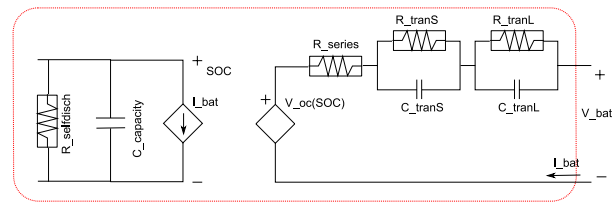

Figure 6. Electrical model of the battery.

\subsection{Electronic device}

Finally, the electronic device can be modeled as a first approximation as a resistor of value $V^{2} / P_{c}$, where $P_{c}$ is the rated power consumption of the device. In complex devices there can be several consumption modes, and each of them has a different power consumption. Verilog-A allows the modeling of this complex behavior, changing the value of the resistor depending on specified conditions that mimic the real device behavior.

\section{Simulation examples}

In this work, two examples are considered: a battery-less sensor with an RF transmitter powered with a discontinuous operation profile, and a battery connected to the energy harvesting system that supplies power to the RF transmission module continuously.

We considered the component from Nordic nRF24E1, a microcontroller with an integrated RF transmitter at 1 Mbps [7]. This device needs a $3 \mathrm{~ms}$ phase of configuration time with a power consumption of $5.7 \mathrm{~mW}$, then a short interval ( $0.3 \mathrm{~ms}$ approximately) to access the ADC and RF transmitter, with a power consumption of $27 \mathrm{~mW}$. After that, the chip enters in sleep mode. The Verilog-A model of the Nordic load takes into account this power consumption profile, in such a way that after configuration, the message is repeated 4 times in $40 \mathrm{~ms}$.

\subsection{Battery-less RF transmitter}

In the case of the battery-less transmitter, it is assumed that the sensor is normally completely switched off, not allowing even to operate the device in sleep mode or other low consumption modes. Whenever a predetermined amount of energy has been collected after some time, the device is connected to the power supply system, then draining the storage capacitor until another predetermined voltage level, and then it is again disconnected waiting for a replenishment of the storage capacitor. Figure 7 shows the schematic design of the system to be simulated.

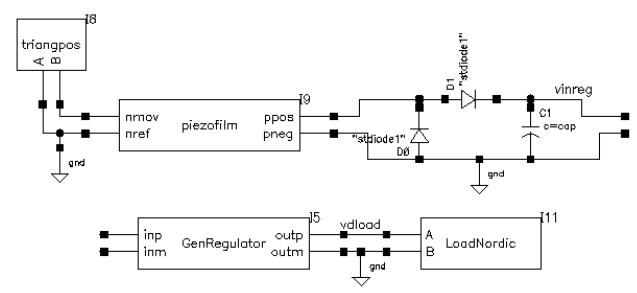

\section{Figure 7. Schematic of a battery-less self-} powered RF transmitter.

The voltage supply is $2 \mathrm{~V}$, and this fact together with the short transmission time has as a result a relatively small storage capacitor $(7 \mu \mathrm{F})$ giving a periodicity of transmission of $11.8 \mathrm{~s}$. This is shown in Figure 8.

\subsection{Battery charger}

For a system including the battery, the device is not restricted to await operation in completely switched off state, but it can be in some of the various low power modes available. While the storage capacitor is below the voltage level needed to activate the regulator, the battery supplies the energy to the device. During the time when the regulator is active, the battery can accept energy coming from the storage 


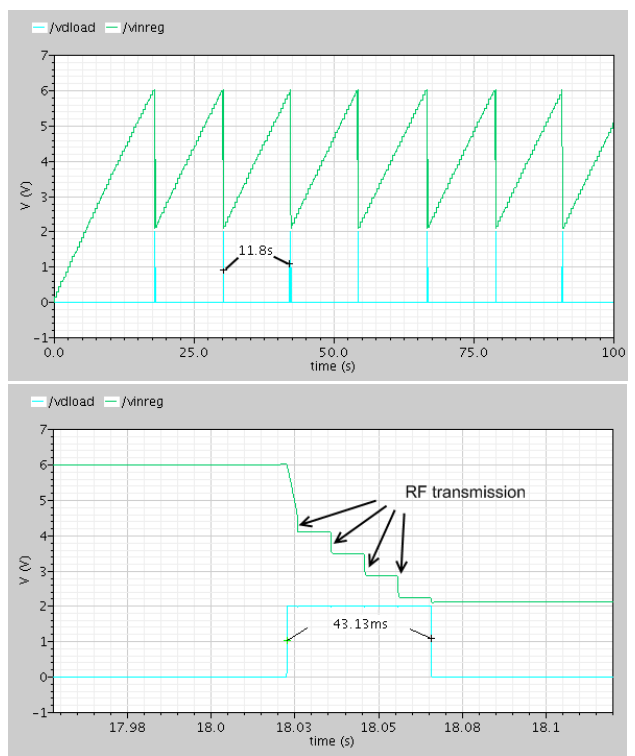

Figure 8. Simulation waveforms of the regulator input voltage, vinreg, and Nordic module voltage supply, vdload.

capacitor via the regulator. A diode avoids discharging the battery through the regulator when it is not active. Figure 9 shows the schematic design of the system to be simulated.

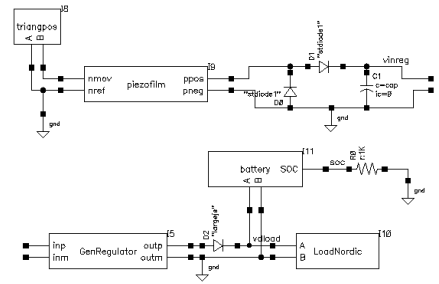

Figure 9. Schematic of a battery-powered RF transmitter.

The employed battery model gives the battery's state of charge (SoC), so that from the simulations it is possible to evaluate if the battery is being charged or discharged. In an energy harvesting application, it is desirable to have energy neutral operation [3], that means, to ensure that the energy harvesting transducer is generating at least the same amount of energy as the energy consumed by the electric load. In other words, the charge transferred during recharge should exceed that which was lost during the off times. This can be expressed as a relation between average currents:

$$
\left\langle I_{r e g}\right\rangle=\left\langle I_{\text {load }}\right\rangle+\left\langle I_{\text {gnd }}\right\rangle
$$

where $\left\langle I_{r e g}\right\rangle$ is the average current flowing into the voltage regulator, $\left\langle I_{\text {load }}\right\rangle$ is the average current consumption of the load, and $\left\langle I_{g n d}\right\rangle$ is the average current of the ground current lost through the regulator and voltage monitoring circuitry.

The expression for $\left\langle I_{\text {load }}\right\rangle$ is:

$$
\left\langle I_{\text {load }}\right\rangle=I_{\text {sleep }}+\frac{t_{R F}}{T_{R F}}\left(I_{R F}-I_{\text {sleep }}\right)
$$

where $I_{\text {sleep }}$ is the current consumption of the communication module in sleep mode, $t_{R F}$ is the time needed to transmit a packet data, $T_{R F}$ is the period between transmissions and $I_{R F}$ is the current consumed during a transmission. Depending on the value of the transmission period, $T_{R F}$, the average current consumed by the load can be calculated, giving a value always greater than $3.16 \mu \mathrm{A}$ which corresponds to the sleep mode current consumption.

On the other hand, the regulator gives an average current that can be calculated as:

$$
\left\langle I_{r e g}\right\rangle=I_{o n} \frac{t_{o n}}{T_{o n}}
$$

where $I_{o n}$ is the input current of the regulator, $t_{o n}$ is the time in which the regulator is activated and $T_{o n}$ is the period between activations. $I_{o n}$ depends on the diode forward voltage, that can only be increased through increasing the regulator output voltage (the diode's cathode voltage is fixed by the battery). The ratio $t_{o n} / T_{o n}$ can be increased especially by increasing the number of piezoelectric films, so that the regulator switches on more often. Both $t_{o n}$ and $T_{o n}$ depend on the product $C_{s}\left(V_{\text {lim }}-V_{\text {reg }}\right)$ so that its ratio remains essentially constant by changing the storage capacitor, $C_{s}$, or the voltage drop, $\left(V_{\text {lim }}-V_{\text {reg }}\right)$.

Figure 10 shows the simulation results of the voltage waveforms and the state of charge of the battery for convenient values of storage capacitor $(10 \mu \mathrm{F})$ and number of devices (10). Table 1 shows the results for other configurations. It is observed how a smaller number of devices do not provide enough average current, and therefore are not able to work in energy neutral operation.

\begin{tabular}{|c|ccc|}
\hline $\mathrm{n}$ & $I_{\text {reg }}(\mu \mathrm{A})$ & $I_{\text {load }}(\mu \mathrm{A})$ & $S o C$ \\
\hline 5 & 1.957 & 3.364 & 0.761 \\
7 & 2.775 & 3.369 & 0.783 \\
10 & 3.964 & 3.378 & 0.816 \\
\hline
\end{tabular}

Table 1. Average currents and final state of charge after $100 \mathrm{~s}$ operation for several systems with different number of parallel piezoelectric elements, $n$. Initial state of charge is 0.8. The transmission period, $T_{R F}$, is fixed at $30 \mathrm{~s}$. Storage capacitor, $C_{s}$, is $10 \mu \mathrm{F}$. 


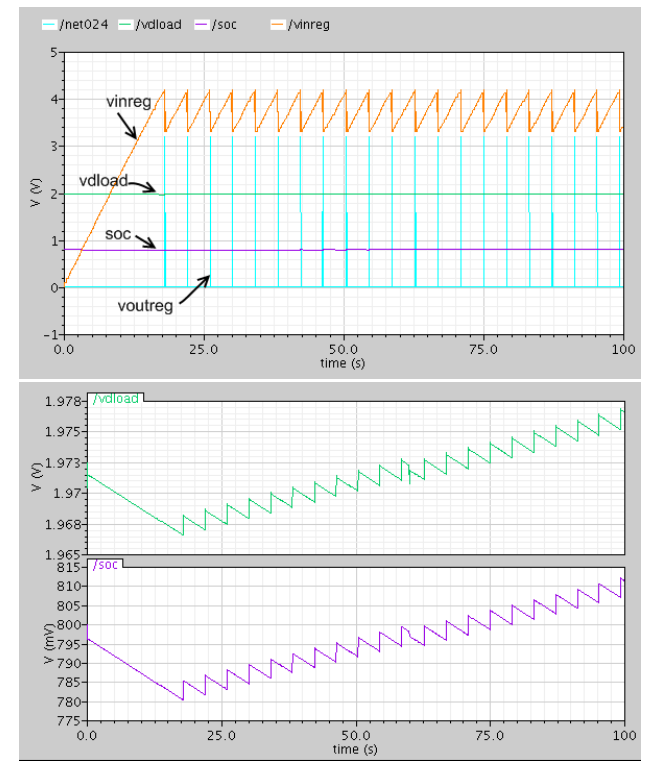

Figure 10. Simulation of voltage waveforms at input and output of the regulator (top). Waveforms of battery voltage and state of charge (SOC) (bottom).

\section{Conclusions}

It was here presented a simulation environment based on Verilog-A that allows to assess the feasibility of a selfpowered electronic device based on a mechanical energy harvesting generator and make the necessary adjustments in the electrical and mechanical part of the system to accomplish the desired objectives.

Two configurations were studied: a battery-less device in which the RF communication module is powered intermittently, and a more complex system where the RF module is powered continuously aided by a battery. The Verilog-A model allows to predict the time between transmissions.

It was shown that the battery-less alternative is easier to accomplish. This is a useful alternative for such applications where the system does not require to make any operation in between transmission events, and there is not a requirement for a minimum fixed transmission periodicity.

On the other hand, the battery allows to maintain operation of the device, at the cost of a higher average consumption current. In order to have a positive energetic balance and thus allowing a recharge of the battery, the energy harvesting device needs to compensate the average current.

\section{Acknowledgments}

This research work has been partially supported by the FUTURIC project of the Spanish Ministry of Science and Education, TEC2005/02739. Loreto Mateu wishes to acknowledge the UPC-Recerca grant from UPC.

\section{References}

[1] Tima 2006 annual report, March 2007.

[2] M. Chen and G. Rincon-Mora. Accurate electrical battery model capable of predicting runtime and I-V performance. Energy Conversion, IEEE Transactions on, 21(2):504-511, 2006.

[3] A. Kansal, D. Potter, and M. Srivastava. Performance aware tasking for environmentally powered sensor networks. In Joint International Conference on Measurement and Modeling of Computer Systems, 2004.

[4] J. Kymissis, C. Kendall, J. Paradiso, and N. Gershenfeld. Parasitic power harvesting in shoes. In IEEE 2nd Intl Conf on Wearable Computing, 1998.

[5] L. Mateu and F. Moll. Optimum piezoelectric bending beam structures for energy harvesting using shoe inserts. JOURNAL OF INTELLIGENT MATERIAL SYSTEMS AND STRUCTURES, 16(October):835-846, 2005.

[6] L. Mateu and F. Moll. Appropriate charge control of the storage capacitor in a piezoelectric energy harvesting device for discontinuous load operation. Sensors And Actuators APhysical, 132(1):302-310, Nov 82006.

[7] Nordic. nRF24E1. 2.4GHz RF transceiver with embedded 8051 compatible microcontroller and 9 input, 10 bit ADC. Technical specification, March 2006.

[8] Open Verilog International. Verilog-A Language Reference Manual. Analog Extensions to Verilog HDL, version 1.0 edition, 1996.

[9] T. Starner. Human-powered Wearable Computing. IBM Systems Journal, 35(3\&4), 1996.

[10] T. Starner and J. Paradiso. Human generated power for mobile electronics. In C. Piguet, editor, Low Power Electronics Design, chapter 45. CRC Press, 2004.

[11] T. Zimmerman. Personal area networks: near-field intrabody communication. IBM Systems J., 35(3 \& 4):609-617, 1996. 